\title{
Exploring Risk Factors with Crash Severity on China Two-Lane Rural Roads Using a Random-Parameter Ordered Probit Model
}

\author{
Shikun Xie $\mathbb{D}$, , ${ }^{1,2}$ Xiaofeng Ji $\mathbb{D},{ }^{1}$ Wenchen Yang $\mathbb{D},^{2}$ Rui Fang $\mathbb{D},{ }^{2}$ and Jingjing Hao $\mathbb{D}^{3}$ \\ ${ }^{1}$ Faculty of Transportation Engineering, Kunming University of Science and Technology, Kunming 650504, China \\ ${ }^{2}$ National Engineering Laboratory for Surface Transportation Weather Impacts Prevention, \\ Broadvision Engineering Consultants, Kunming 650200, China \\ ${ }^{3}$ School of Transportation and Logistics, Southwest Jiaotong University, Chengdu 610031, China \\ Correspondence should be addressed to Xiaofeng Ji; yiluxinshi@sina.com
}

Received 23 July 2020; Revised 8 November 2020; Accepted 30 November 2020; Published 17 December 2020

Academic Editor: Eleonora Papadimitriou

Copyright (C) 2020 Shikun Xie et al. This is an open access article distributed under the Creative Commons Attribution License, which permits unrestricted use, distribution, and reproduction in any medium, provided the original work is properly cited.

\begin{abstract}
Understanding the factors that contribute to traffic crashes can help provide a fundamental basis to plan and develop appropriate countermeasures for road safety issues emerging in particular on two-lane rural roads. However, most of the studies have focused on urban roadways and freeway systems, and few studies have investigated the issue of heterogeneity on two-lane rural roads. The purpose of this study is to uncover the risk factors influencing crash severity on two-lane rural roads in China. A sample of 1490 traffic crashes occurring on two-lane rural roads between 2012 and 2017 was collected from the Mouding County Highway Bureau in Yunnan, China. A random-parameter ordered probit model was estimated using these data to capture underlying unobserved characteristics in personal traits, vehicle attributes, roadway conditions, environmental factors, and crash attribute. To better understand the effect of critical factors on crash severity outcome probability, an elasticity analysis was then introduced. The results show that six factors such as driver's attribution, illegal driving behaviour, access segment, day of week, vehicle type, and crash form have a significant impact on the injury severity, and the impacts of driving behaviours, access segment, and vehiclefixed object crashes had significant variation across observations. Besides, the correlations between critical factors and the probability of serious injury sustained in traffic crashes are identified and discussed. The local driver indicator has more positive impact on the crash severity than nonlocal driver, and nonaccess segment appears a higher probability of serious or vicious collisions. It is worth mentioning that motorcycle-involved crashes do show an obvious correlation with crash injury severity. As for crash forms, vehicle-vehicle crashes are more likely to lead to severe crash injury. Besides, high-risk driving behaviour (e.g., fatigue driving, speeding, and converse driving), weekends, and holidays are found to have significant contribution to increasing the probability of traffic crash injuries and fatalities on two-lane rural roads.
\end{abstract}

\section{Introduction}

Two-lane roads constitute a major portion of the rural road system in most countries of the world. However, due to the limited roadside protective facilities, complex terrain conditions, and roadway geometric features, mixed traffic flows involving trucks and motorcycles on two-lane rural roads impose significant safety issues and high crash rates. In China, 114,287 traffic crashes occurring on two-lane rural roads resulted in nearly $71 \%$ of the total fatalities on highways in 2013 [1]. In Europe, approximately $60 \%$ of road accident fatalities occur on two-lane rural roads [2]. In addition, the National Highway Traffic Safety Administration (NHTSA) reported that rural areas held only $19 \%$ of the total U.S. population but induced $48 \%$ of fatal traffic crashes and $49 \%$ of traffic fatalities in 2015 [3]. There is a need to investigate why higher proportions of fatal and severe injuries are occurring on two-lane rural roads to develop effective countermeasures for improving traffic safety in rural areas.

For the two-lane rural road, it is significantly different from two-lane rural highways. Two-lane rural road is the key road connecting country town and counties, and two-lane rural highway is a passage between counties. The most 
obvious difference is the lack of central dividers and roadside isolation facilities on two-lane rural roads. At the same time, the two-lane rural road is a most transportation channel, especially for underdeveloped areas. The two-lane rural roads are limited by terrain conditions and funds, and the technical indicators of road sections are low. There are many road sections along the cliff and river, large slopes, small turning radius, and insufficient sight distance, and it contains a large number of bridges and tunnels, intersections, and villages, with complicated road conditions. In addition, because the roads are mostly used by trucks and motorcycles, the vehicle load is large, and the risk factor is high, higher proportions of fatal and severe injuries occur on two-lane roads, which directly affects the economic benefits of rural areas and brings many adverse effects to regional development.

Unsurprisingly, much attention and effort has been paid to exploring the relationships between different collections of risk factors and crashes from various roadway entities, with the major focus on highways $[4,5]$, urban roads [6], intersections [7], and tunnels [8]. For instance, Chiou et al. [9] took Taipei City as an example and modelled and analysed the severity of two-car collision accidents at intersections. They found that the collision type, collision location, and driver characteristics contribute to severe intersection accidents. Yu and Abdel-Aty [10] studied collision accidents on expressways and found that rapid change and low visibility are the key factors that increase the severity of crashes. Compared with urban roadway and freeway systems, rural roadway system is more concentrated with fatal traffic crash injuries and fatalities due to complex terrain and road geometry constraints [11]. Existing research has conducted preliminary studies on the identification of factors that affect the crash severity on the rural roads. However, it is limited, and one specific direction that seems to be missing in the current two-lane rural road crash literature is the issue of heterogeneity.

Heterogeneity has significant implications on safety countermeasure development and program design since it may affect the accuracy and consistency of parameter estimation and crash predictions [12]. It is reasonable to assume that the occurrence of a collision is a complex process, caused by the interaction of multiple factors in personal traits, vehicle attributes, roadway conditions, and the environmental factors. However, some of the factors (e.g., collision speed) associated with the severity of crashes are not observed or nearly impossible to collect in collision investigations, and the missing influence factors become the heterogeneity source of the analysis results. For example, the traditional analysis model sets the influence of the driver's age in the same group on the severity of the collision to be the same when analysing the influence of the driver's age on the severity of the collision. In fact, drivers have significant differences in brain development, driving skills, and perceived risk, even in the same age group (for example, younger than 25 years old). Due to the unobservability of these data, the differences of these factors are ignored in analysis. Therefore, ignoring the possible heterogeneity in the crash data will lead to the biased parameters could be estimated, and incorrect inference could be drawn [13]. Many research methods to analyse crash severity have been used in previous studies, but statistical modelling has been the primary method. Because the dependent variables of crash severity models are typically binary or multicategory outcomes [14], statistical analysis can select models based on the categories of dependent variables. Therefore, logit models have been widely used $[15,16]$. Crash severity was found to be of different grades, namely, minor injuries, serious injuries, and deaths. The higher the grade, the more serious the crash. Moreover, there is not a simple nonlinear relationship between the severity of the accident and the influencing factors, so the ordered probability model has gradually been used to analyse the factors that affect crash severity in most areas. For instance, Michalaki et al. [17] used a generalized ordered logistic regression model to determine the factors that affect the severity of hard shoulder and main road collisions. Lemp et al. [18] applied a mixed-order probit model to study the effects of vehicle, occupant, driver, and environmental characteristics on the severity of heavy truck crashes. It can clearly be seen that existing studies have revealed that ordered response models explicitly account for the inherent ordering within the response variable, while unordered response models do not recognize the ordering [19]. The ordered probability model has a stronger explanatory power, and the severity of traffic accidents is an orderly output variable, so most studies rely on the ordered probability model to study the severity of traffic accidents [20].

Since the crash severities are inherently ordered, the ordinal model is preferred to appropriately represent the relationship between the ordinal nature of response outcomes and independent variables. One limitation imposed by the traditional discrete choice approach (including the standard multinomial and ordered logit models) when applied to the crash injury data is that it cannot allow for cross-individual heterogeneity. In reality, as each individual being analysed has specific characteristics that may influence the severity outcomes differentially, the impacts of explanatory variables are expected to vary across observation. Recently, the random-parameter model that considers heterogeneity becomes increasingly prevalent among traffic safety professionals and has been demonstrated superior with a substantially improved goodness-of-fit and the ability to address the heterogeneous effects due to unobserved factors. For instance, Wu et al. [21] developed mixed logit models to analyse driver injury severities in single-vehicle (SV) and multivehicle (MV) crashes on rural two-lane highways in New Mexico from 2010 to 2011 and concluded that vehicle-fixed object crashes have heterogeneity effect on the severity of crash. Zhou et al. [22] established a randomparameter logistic model to estimate the likelihood of fatal and severe injuries to passengers as a function of various factors and indicated substantial inconsistences in the effects of risk factors between models of noncollision injuries and collision injuries. Chen el al. [23] considered the unobserved heterogeneity of parameter effects, developed a randomparameter bivariate ordered probit model to examine factors affecting injury sustained by two drivers involved in the 
same rear-end crash between passenger cars, and found that the proposed model outperforms the two separate ordered probit models with fixed parameters.

In doing so, to capture the heterogeneity across different crashes, a random-parameter ordered probit model was developed to identify significant factors and quantify their impacts on two-lane rural road crash injury severities. This study differs from existing studies by simultaneously considering the following aspects: on the one hand, to conduct a quantitative study on the severity of two-lane rural road crashes by comprehensively considering the heterogeneity of drivers, vehicles, rural road characteristics, and driving environment from a comprehensive and integrated perspective, the characteristics of the two-lane rural road are taken into account, including access segment, road sections through villages and towns, and motorcycles. On the other hand, in-depth study of the impact of various indicators on the crash severity of two-lane rural road was conducted, and the influence mechanism of significant variables was obtained. This study seeks to provide an insightful understanding of the effects of these contributing factors on the frequency and seriousness of crashes to aid government agencies and traffic engineers in designing and developing proactive road safety countermeasures for severe injury prevention. The protentional contributions of this study are summarized as follows: (1) this study can be regarded as an initial effort to reveal the significant factors that affect crash severity on two-lane rural roads, and traffic safety can be improved by controlling and/or improving these factors. (2) This study focuses on two-lane rural roads, and the significant influencing factors obtained can expand the perspective of two-lane rural road traffic safety research.

\section{Materials}

2.1. Study Area. China's terrain is complex and diverse, and mountainous areas account for $69.1 \%$ of the country. Rural roads play an irreplaceable role in mountainous areas and are the infrastructure to promote the development of villages and towns. However, due to geological terrain conditions, the geometric alignment of two-lane rural roads is extremely complex, which makes drivers likely and rapidly accelerate, sharply decelerate or turn, or perform other high-risk driving behaviours. The driving environment of a two-lane rural road is shown in Figure 1.

In this study, the two-lane rural road is located in the north-central part of the plateau in Yunnan Province, China, which is $85 \mathrm{~km}$ in length and $8.5 \mathrm{~m}$ in width. The design speed is $60 \mathrm{~km} / \mathrm{h}$, while the limit speed is $80 \mathrm{~km} / \mathrm{h}$. It is a twoway road with modified asphalt concrete pavement and the absence of centre dividers. In terms of road design, the minimum radius of the flat curve is 125 metres, and the longitudinal slope is $6 \%$. The accumulated ratios in the length of the village segment, access section, curve, uphill and downhill section, bridge, and tunnel are greater than $90 \%$, and the percentage of bridges and tunnels in the total mileage is $17 \%$. The driving environment is extremely complicated and is considered typical two-lane rural roads. On average, there has been a crash every month. Therefore, it is known as the "Death Road" and "Devil Road" in the local area. In order to better illustrate the location of this two-lane rural road of interest, a high-resolution map is shown in Figure 2.

2.2. Data Collection. To analyse the crash severity of twolane rural roads, we collected crash data from the Mouding County Highway Bureau, and the crash file contains the crash number, time, location, vehicle type, weather condition, passenger information, driver information, crash severity, and other environmental variables. At the same time, driving environment data and road line design data were collected. The road file contains information about the type of road, the number of lanes, shoulder type, corners, flat curve radius, slope, intermediate belt, shoulder width, and other related geometric features. The driving environment data are obtained from actual research, including whether the road has intersection, the type of the intersection, the slope of the intersection, the angle, and the material of the branch road surface.

Crashes for a two-lane rural road from 2012-2017 were obtained, including a total of 2247 crash data points that occurred. To ensure the correctness of the analysis results, the original data were processed, the rows containing empty data points were removed, the crash data were encoded and classified, and the driving environment data and road line data are matched according to the location of the crash. Finally, 1,490 crash data points with complete information were constructed into a rural road dataset.

\subsection{Variable Selection}

2.3.1. Independent Variables. According to the road alignment and crash information of the two-lane rural road, 14 potentially influencing factors were selected from five aspects: driver, vehicle, road, environment, and crash. Among them, the driver attributes include driver gender, age, attribution, and driving behaviour in the crash; vehicle characteristics include the composition of the crash vehicle; road attributes include horizontal alignment, vertical alignment, access section, and village segment; driving environment attributes include light, weather, road surface, and the time of weekday; and the collision attribute is the form of the accident.

Considering the structural characteristics of different road operating environments and their impact on the driver's driving behaviour combined with the actual characteristics of two-lane rural roads through the village, isolation measures on both sides are lacking. Two characteristic indexes of the severity of collisions on rural roads are put forward, namely, the road access segment and the village segment. Currently, there is no clear definition of the access segment and the crossing section of the village segment. In foreign countries, roads are usually divided into two types: road sections and intersections. Crashes in the $76 \mathrm{~m}$ range of intersections are identified as intersection crashes, and the rest are road section crashes [24]. In the country, the roads are generally divided into the range of villages and towns 


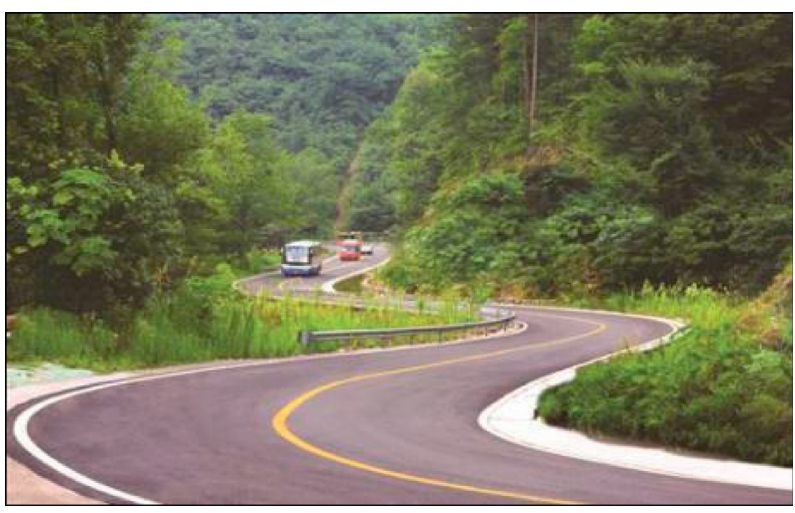

(a)

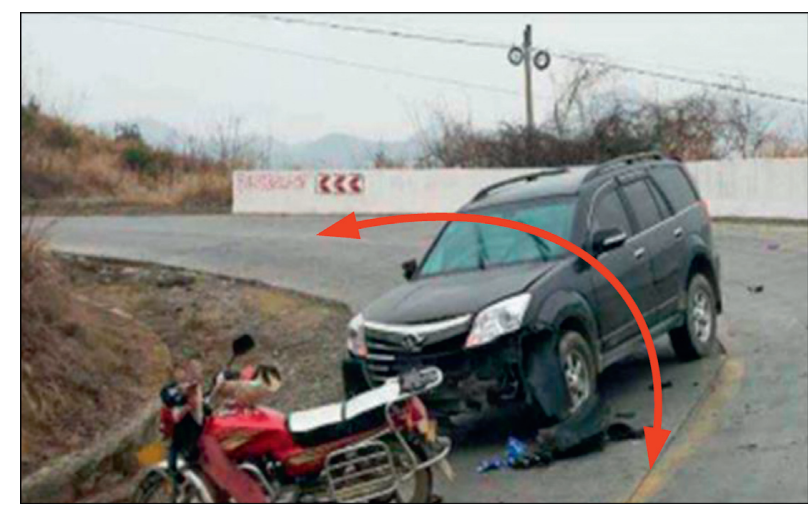

(b)

FIgURE 1: Driving environment of two-lane rural roads.

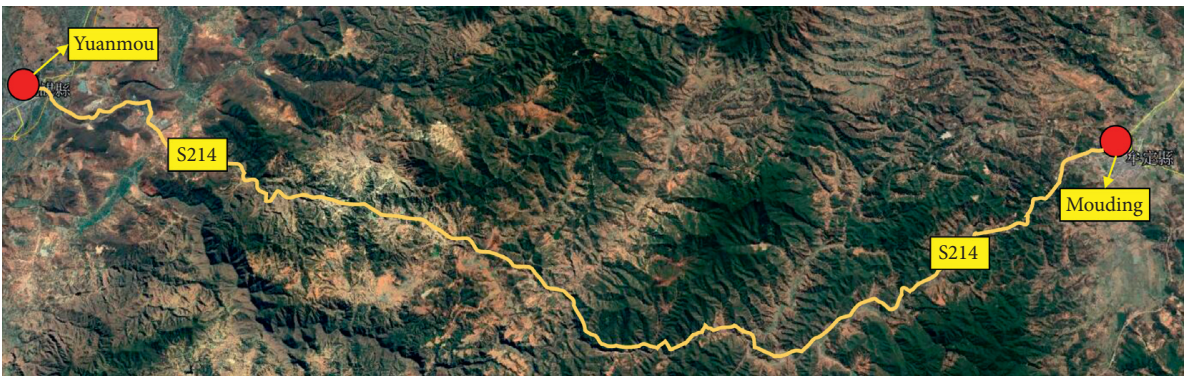

Figure 2: The location of the two-lane rural road.

through the extension of 100 metres on both sides of actual villages and towns [25]. Therefore, the section of the access segment is defined as the actual intersection and the length of $76 \mathrm{~m}$ on both sides; the section of the road through the village is the actual village (the location is based on the village and town signs) and each side of the 100-metre extension. The schematic diagrams of access segment and village segment are shown in Figure 3, respectively.

2.3.2. Definition of Crash Severity. According to the Law of the People's Republic of China on Road Traffic Safety in 2004, China has classified the severity of crash injury severity into four levels according to the degree and amount of personal injury or property damage [19], namely, death, serious injury, minor injury, and property loss. Therefore, with reference to the standards of the Ministry of Public Security, the combination of collision caused by crashes and property losses, the crash severity was divided into three levels, minor collision, serious collision, and malignant collision, as follows (see Table 1 for details):

Minor collision: no person was injured.

Serious collision: person was injured immediately, and the number of injuries (including minor or serious injuries) was less than 3 , but no person was killed.

Malignant collision: person was killed immediately or the number of injuries (including minor or serious injuries) was more than 3 in the vehicle collision.
Table 2 shows the definitions and descriptive statistics of the explanatory variables for the empirical analysis.

\section{Methods}

In this study, considering the issue of heterogeneity on twolane rural roads and following the present methodology [26], a random-parameter ordered probit model is developed to identify significant casual factors and quantify their impacts on crash injury severity in all collisions on two-lane rural roads. The proposed analytical framework is summarized in Figure 4. In detail, this study collected data from crashes, linear design, environment, and traffic flow, and datasets are standardized for processing, including data extraction, processing, coding, and classification to establish a basic database of two-lane rural roads. Then, after the parallel line hypothesis test and the argument variable multilinear test are carried out, a variable impact analysis was then conducted using the random-parameter ordered probit model to investigate the effects of the explanatory variables on injury severity. These findings further provide insights for a better understanding of crash severity on two-lane rural roads. Finally, the estimated coefficients are not sufficient to determine the net change in the outcome probabilities which cause the change in the explanatory variables, and the results are provided by a detailed discussion in conjunction with marginal effects and elasticities. The following subsections present the details of the hypothesis test, the random-parameter ordered probit model parameter tests, and elasticity analysis. 


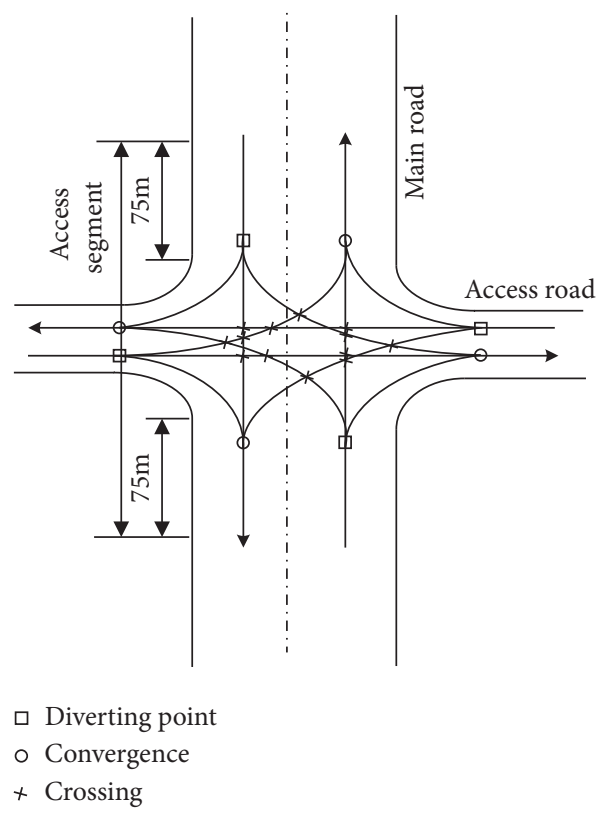

(a)

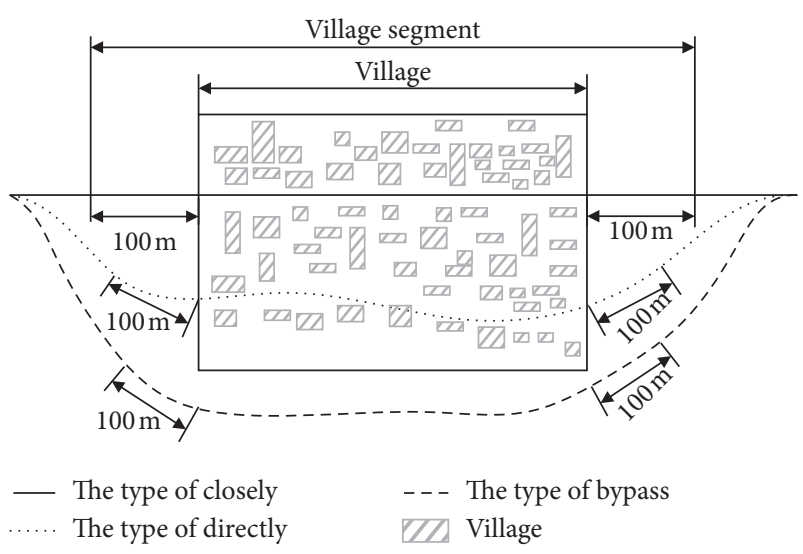

(b)

Figure 3: Schematic diagram of typical section division: (a) access segment and (b) village segment.

TABLE 1: Classification of the seriousness of crashes on two-lane rural roads.

\begin{tabular}{lcc}
\hline Crash severity level & Crash injury severity & Criteria for the classification \\
\hline I & Minor accident & Number of deaths $=0$, number of injured $=0$ \\
II & Serious accident & Number of deaths $=0$, number of injured $=1 \sim 3$ \\
III & Malignant accident & Number of deaths $>1$, number of injured $>3$ \\
\hline
\end{tabular}

\subsection{Hypothesis Tests}

3.1.1. Multicollinearity Hypothesis Test. No multicollinearity between variables is a prerequisite for ordered probabilistic regression models. In other words, if there is a strong correlation between variables, the basic assumption is violated and the ordered regression model cannot be constructed. In this study, the method of variance inflation factor (VIF) is used to diagnose collinearity among variables [27]. Generally, tolerance is less than 0.1 or VIF is greater than 10 and is collinear. The variable collinearity test was performed by stata15.0, and Table 3 shows the results; all variables had more than 0.1 tolerances and less than 10 VIF, so there was no multicollinearity.

3.1.2. Parallel Line Hypothesis Test. The preconditions of the ordered regression model require that the spacing between the classifications under the dependent variables must be equal [9]. That is, the level of the incremental gap between minor collisions and serious collisions and between serious collisions and malignant collisions should be equal; otherwise, the analysis results of the ordinal regression model are not accurate, and parameter estimates will fail, resulting in unreliable models. Therefore, to construct an ordered regression model, we first need to test the parallel line hypothesis of the model. In this paper, the Wald test is used to test the parallel line hypothesis. Table 4 shows the results, the chi-square value is 14.206 , and the
$P$ value is 0.170 (greater than 0.05 ), which means that no explanatory variables violated the parallel regression assumption. That is, the regression equations of different levels of severity are parallel to each other, and the ordinal model could fully fit the crash severity of two-lane rural roads.

\subsection{Random-Parameter Ordered Probit Model.} Random-parameter ordered probit model specification starts from the standard ordered probability model, which captures the effect of independent variables on the ordinalnatured response variable representing injury severity levels. This is derived from a latent variable $y^{*}$ and specified as a linear function for each crash observation, such that

$$
y^{*}=X \beta+\varepsilon,
$$

where $X$ is explanatory variables, $\beta$ is coefficient vector, and $\varepsilon$ is the error term that assumed to obey the standard normal distribution. Taking equation (1), each crash severity outcome can be expressed as the following equation:

$$
\begin{array}{ll}
y=0 \text { if } & y^{*} \leq 0, \\
y=1 \text { if } & 0<y^{*} \leq \mu_{1}, \\
y=2 \text { if } & \mu_{2}<y^{*} \leq \mu_{1}, \\
\ldots & \\
y=0 \text { if } & \mu_{j-1} \leq y^{*},
\end{array}
$$


TABLE 2: Descriptive statistics of explanatory variables for analysing two-lane rural road crash severity.

\begin{tabular}{|c|c|c|c|}
\hline Variables & Description & $\begin{array}{l}\text { Frequency/ } \\
\text { average }\end{array}$ & $\begin{array}{l}\text { Percentage/ } \\
\quad \text { SD }\end{array}$ \\
\hline \multicolumn{4}{|l|}{ Accident attributes } \\
\hline \multirow{4}{*}{ Form of collision (CR_FORM) } & 1 if the collision belongs to single-vehicle accident (CR_FORM1) & 142 & $9.53 \%$ \\
\hline & $\begin{array}{l}2 \text { if the collision belongs to vehicle-vehicle crash vehicle } \\
\text { (CR_FORM2) }\end{array}$ & 1152 & $77.32 \%$ \\
\hline & $\begin{array}{c}3 \text { if the collision belongs to vehicle-fixed object crashes } \\
\text { (CR_FORM3) }\end{array}$ & 99 & $6.64 \%$ \\
\hline & $\begin{array}{l}4 \text { if the collision belongs to vehicle-pedestrian crashes } \\
\text { (CR_FORM4) }\end{array}$ & 97 & $6.51 \%$ \\
\hline \multicolumn{4}{|l|}{ Driver attributes } \\
\hline \multirow{2}{*}{ Driver gender (DR_GE) } & 0 if male & 1294 & $86.85 \%$ \\
\hline & 1 if female & 195 & $13.09 \%$ \\
\hline Driver age (DR_AG) & - & $16 \sim 65$ & 9.5 \\
\hline \multirow{2}{*}{ Local driver (DR_LO) } & 0 if true & 1056 & $70.87 \%$ \\
\hline & 1 otherwise & 434 & $29.13 \%$ \\
\hline \multirow{2}{*}{ Behaviour of driver (DR_BE) } & 0 if exist illegal driving behaviours & 835 & $56.04 \%$ \\
\hline & 1 otherwise & 655 & $43.96 \%$ \\
\hline \multicolumn{4}{|l|}{ Vehicle attributes } \\
\hline \multirow{3}{*}{ Type of vehicle (VEH_TYPE) } & 1 if the collision only involved passenger cars (VEH_TYPE1) & 1054 & $70.74 \%$ \\
\hline & 2 if the collision related to motorcycle (VEH_TYPE2) & 315 & $21.14 \%$ \\
\hline & 3 if the collision regard with motorcycles (VEH_TYPE3) & 121 & $8.12 \%$ \\
\hline \multicolumn{4}{|l|}{ Road attributes } \\
\hline \multirow{2}{*}{$\begin{array}{l}\text { Road horizontal alignment } \\
\text { (RD_HA) }\end{array}$} & 0 otherwise & 641 & $43.02 \%$ \\
\hline & 1 if curve & 849 & $56.98 \%$ \\
\hline \multirow{2}{*}{ Road vertical alignment (RD_VA) } & 0 otherwise & 926 & $62.15 \%$ \\
\hline & 1 if downhill or uphill & 564 & $37.85 \%$ \\
\hline \multirow{2}{*}{ Access segment (RD_AS) } & 0 if access segment & 1100 & $73.83 \%$ \\
\hline & 1 otherwise & 390 & $26.17 \%$ \\
\hline \multirow{2}{*}{ Village segment (RD_VS) } & 0 if village segment & 915 & $61.41 \%$ \\
\hline & 1 otherwise & 575 & $38.59 \%$ \\
\hline \multirow{2}{*}{ The surface of road (RD_SU) } & 0 otherwise & 1420 & $95.30 \%$ \\
\hline & 1 if wet, icy, and snowy & 70 & $4.70 \%$ \\
\hline \multicolumn{4}{|l|}{ Environment attributes } \\
\hline \multirow{2}{*}{ The light of crash (EN_LA) } & 0 otherwise & 967 & $64.90 \%$ \\
\hline & 1 if light poor & 513 & $34.43 \%$ \\
\hline \multirow{2}{*}{ The weather of crash (EN_WA) } & 0 otherwise & 1391 & $93.36 \%$ \\
\hline & 1 if rain, snow and fog & 99 & $6.64 \%$ \\
\hline \multirow{2}{*}{ The day of weekday (EN_TA) } & 0 otherwise & 1040 & $69.80 \%$ \\
\hline & 1 if weekend or holiday & 450 & $30.20 \%$ \\
\hline
\end{tabular}

where the classification of $y$ is determined by the threshold parameters $\mu . j$ is the highest ordered value. In this study, there are 3 levels of injury severity, $j=0$ for minor collision, $j=1$ for serious collision, and $j=2$ for malignant collision. According to the current study, the highest integer is defined as malignant collision. Assuming that $\varepsilon$ follows a standard normal distribution, the probabilities of $j$ can be expressed by the equation

$$
\begin{gathered}
\operatorname{prob}(y=0 \mid X=\Phi(-\beta X), \\
\operatorname{prob}\left(y=1 \mid X=\Phi\left(\mu_{1}-\beta X\right)-\Phi(-\beta X),\right) \\
\operatorname{prob}\left(y=2 \mid X=\Phi\left(\mu_{2}-\beta X\right)-\Phi\left(\mu_{1}-\beta X\right),\right. \\
\ldots \\
\operatorname{prob}\left(y=j \mid X=1-\Phi\left(\mu_{j-1}-\beta X\right) .\right.
\end{gathered}
$$

There may be certain random effects in each influencing factor. In order to catch invisible heterogeneity of each explanatory variable in crash data, the ordered probit model is extended to include estimation of random-parameter equation (4) which indicates the random effect of observable variables:

$$
\beta_{q}=\beta+\varphi_{q}
$$

where $\varphi_{q}$ is a random error term vector which obeys normal distribution. $\beta_{q}$ is estimated by the maximum likelihood method with Halton draws [28].

3.3. Elasticity Analysis. To assess the effect of significant variables estimated in the random-parameter ordered probit model on crash severity outcome probability, elasticity 


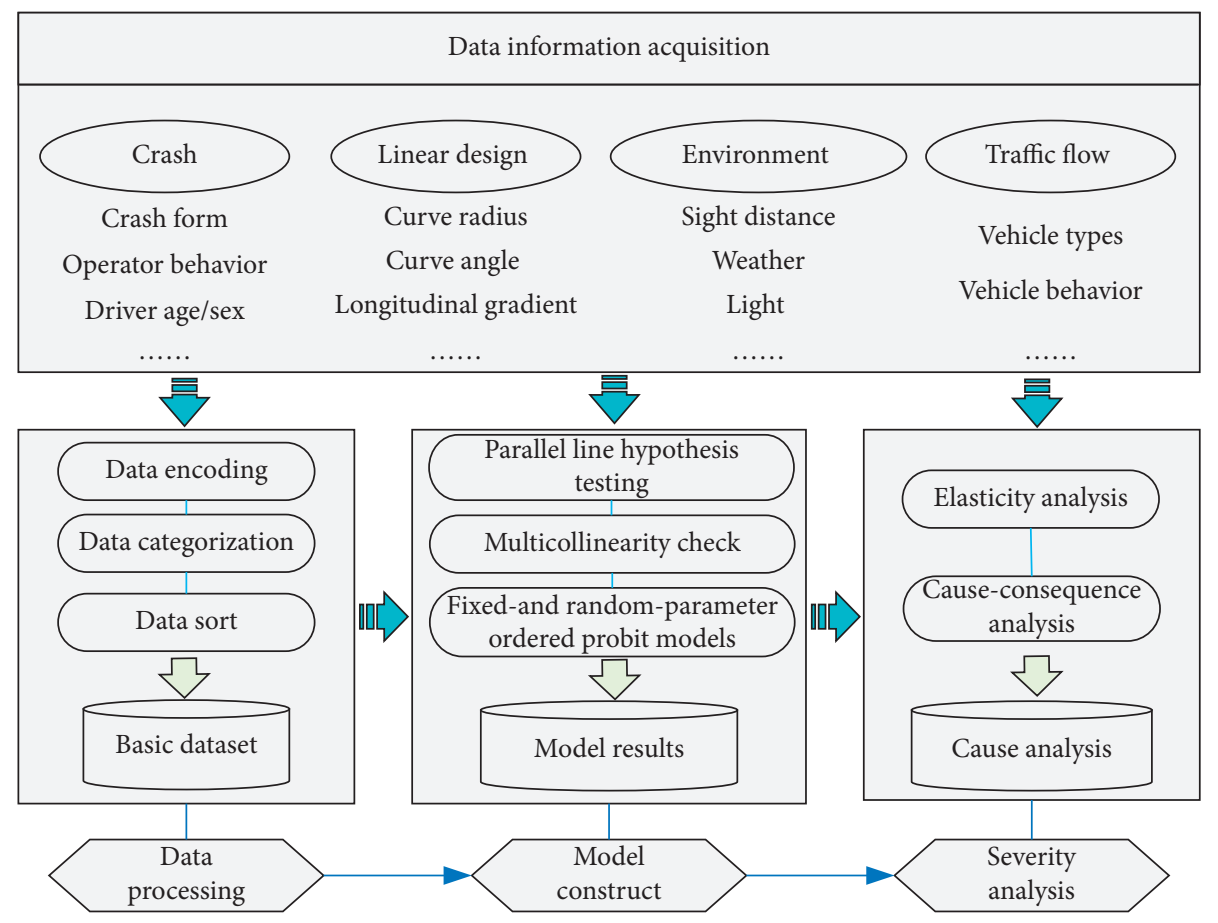

Figure 4: Study framework layout.

TABLE 3: Multicollinearity test variables.

\begin{tabular}{|c|c|c|c|c|}
\hline \multirow[t]{2}{*}{ Variables } & \multirow{2}{*}{$\begin{array}{l}\text { Standardized } \\
\text { coefficients }\end{array}$} & \multirow[t]{2}{*}{ T-stat } & \multicolumn{2}{|c|}{$\begin{array}{c}\text { Collinearity } \\
\text { statistic }\end{array}$} \\
\hline & & & Tolerance & VIF \\
\hline DR_GE & 0.021 & 0.53 & 0.969 & 1.032 \\
\hline DR_AG & 0.002 & 1.34 & 0.961 & 1.041 \\
\hline DR_LO & -0.088 & -2.98 & 0.954 & 1.048 \\
\hline DR_BE & -0.121 & -3.97 & 0.750 & 1.333 \\
\hline RD_AL & -0.038 & -1.37 & 0.915 & 1.093 \\
\hline RD_LS & 0.026 & 0.92 & 0.932 & 1.073 \\
\hline RD_AC & -0.058 & -1.88 & 0.929 & 1.076 \\
\hline RD_VI & -0.008 & -0.28 & 0.932 & 1.073 \\
\hline RD_SU & -0.020 & -0.23 & 0.510 & 1.962 \\
\hline EN_LA & -0.010 & -0.34 & 0.980 & 1.020 \\
\hline EN_WA & -0.078 & -1.06 & 0.512 & 1.952 \\
\hline EN_TA & -0.076 & -2.65 & 0.987 & 1.014 \\
\hline CR_FORM1 & 0.543 & 5.72 & 0.222 & 4.510 \\
\hline CR_FORM2 & 0.462 & 5.32 & 0.130 & 7.679 \\
\hline CR_FORM3 & 0.253 & 2.56 & 0.283 & 6.535 \\
\hline CR_FORM4 & 0.948 & 8.67 & 0.368 & 2.721 \\
\hline VEH_TYPE1 & -0.339 & -6.34 & 0.291 & 3.433 \\
\hline VEH_TYPE2 & -0.293 & -5.04 & 0.307 & 3.257 \\
\hline VEH_TYPE3 & -0.181 & -3.16 & 0.895 & 1.117 \\
\hline
\end{tabular}

TABLE 4: Multicollinearity test variables.

\begin{tabular}{lcccc}
\hline Model & -2log-likelihood & Chi-square & Df & Sig \\
\hline Null hypothesis & 2306.652 & & & \\
General & 2194.452 & 14.206 & 19 & 0.170 \\
\hline
\end{tabular}

analysis is conducted. The purpose is to find variables that have a greater impact on the dependent variable, and some independent variables are statistically significant correlation with the dependent variable. In fact, these variables may have a weaker effect on the dependent variable. Therefore, elasticity analysis is used for comparison to obtain the degree of influence of variable changes on dependent variables. The regular elasticity is computed as [29]

$$
E_{X_{\mathrm{jnk}}}^{P\left(Y_{i}=j\right)}=\frac{\partial P\left(Y_{i}=j\right)}{\partial X_{\mathrm{jnk}}} \cdot \frac{X_{\mathrm{jnk}}}{P\left(Y_{i}=j\right)},
$$

where $X_{\text {jnk }}$ represents the $k$ th explanatory variables associated with crash severity $j$ for collision.

However, it should be noted that elasticities are applicable to continuous variables in other words, if the index variable is a binary classification variable (those with the value of 0 or 1 ), the elastic coefficient cannot be directly calculated by equation (5), since the probability is not differentiable with respect to a value of 0 or 1 . To solve this issue, pseudoelastic coefficients can be computed by calculating the marginal effect of binary variables, as shown in equation (6) [30]. Then, calculate the average direct pseudoelasticity of each injury severity to represent the entire dataset [31]:

$$
E_{X_{\mathrm{jnk}}}^{P\left(Y_{i}=j\right)}=\frac{P\left(Y_{i}=j\right)\left[X_{\mathrm{jnk}}=1\right]-P\left(Y_{i}=j\right)\left[X_{\mathrm{jnk}}=0\right]}{P\left(Y_{i}=j\right)\left[X_{\mathrm{jnk}}=0\right]} .
$$

\section{Results}

4.1. Model Test and Evaluation. To test the overall model fit, used the chi-square distribution and Akaike information criterion (AIC), which are calculated using equations (7) and (8), where the likelihood ratio tests are conducted to 
statistically assess if crash severity models are significantly different across the fixed-parameter model and the randomparameter model:

$$
\begin{aligned}
\chi^{2} & =2\left[L L\left(\beta_{\text {random }}\right)-L L\left(\beta_{\text {fixed }}\right)\right], \\
\mathrm{AIC} & =2 k-2 \ln (L),
\end{aligned}
$$

where the $L L\left(\beta_{\text {random }}\right)$ is the log-likelihood at convergence of the random-parameter ordered probit model and the $L L\left(\beta_{\text {fixed }}\right)$ is the log-likelihood at convergence of the fixedparameter ordered probit model. The likelihood ratio is chisquare distributed with degrees of freedom equal to the difference in the number of parameters of both of the models. $K$ is the number of parameters of the model. The smaller the AIC and the higher the chi-square values, the better the model fits the data.

Table 5 shows the results of goodness-of-fit measures for the models. Undoubtedly, there are substantial differences in goodness-of-fit between fixed-parameter and random-parameter model types when applied to two-lane rural road crash data. In particular, the random-parameter ordered probit model has a log-likelihood of -1066.12 and AIC of 2158.2 (with 17 estimated parameters), which shows improvement compared with -1080.42 and 2198.8 of the fixed-parameter ordered probit model (with 7 estimated parameters). Meanwhile, the likelihood ratio statistic of 28.6 with 10 degrees of freedom denotes that the log-likelihood of the random-parameter model and fixed-parameter model is significantly different at $99.99 \%$ confidence interval. The above results indicate that the random-parameter ordered probit model is superior for modelling two-lane rural roads' crash severity compared with the fixed-parameter ordered probit model. The effects of explanatory variables used in this model are discussed in the following sections.

4.2. The Random-Parameter Ordered Probit Model Result. After quantifying the whole database and optimizing the model, the random-parameter ordered probit model for predicting the crash severity of two-lane rural road was significantly established, and the model parameters were estimated by Nlogit software. The results of the variable estimation effect are presented in Table 6. There are three variables in the model (CR_FORM3, DR_BE and RD_AS) that are normally distributed random parameters. This suggests that the presence of random parameters explicitly demonstrated the existence of heterogeneity in the effects of risk factors.

As illustrated in Table 6, only statistically significant variables have been represented, and the $P$ values of all contribute variables are at a significance level of $90 \%$. According to the parameter estimation results of the random-parameter ordered probit model, a variety of variables are found to significantly influence crash severity outcomes with a total of 6 class variables, and the factors that explain these crashes include DR_LO, DR_BE, RD_AC, EN_TA, CR_FORM, and VEH_TYPE. Note that the predictors with positive coefficient indicate an increase in the probability of occurrence serious or malignant collision as compared to minor collision, while the predictors with negative coefficients mean that these features are the values that may lead to low-level crash severity or even prevent the occurrence of crash injury. According to Table 6 and Figure 5, we can find that the variables that significantly increase the probability of serious or malignant two-lane rural roads are DR_BE (exist illegal driving behaviours), RD_AS (the location of crash) CR_FORM (including the vehicle-vehicle crashes and vehicle-fixed object crashes), and VEH_TYPE (the type of motorcycles). The features that are important to crash severity prediction but reducing the crash severity are as follows: DR_LO (the attribution of driver) and EN_TA (the crash day of the week).

Furthermore, according to the calculation results, the impact of significant variables on the crash severity of China two-lane rural roads is DR_BE $>$ CR_FORM $>$ RD_AS $>$ VEH_TYPE3 $>$ DR_LO $>$ EN_TA. Among them, the regression coefficient of DR_BE is 1.240 , indicating that the driver has dangerous driving behaviours (including retrograde, illegal overtaking, or other illegal driving behaviours) and the probability of serious crashes is greater than normal driving. Then, the two classification variables of crash form, CR_FORM2 and CR_FORM 3, are all at the 90\% significance level, and the regression coefficients of these three variables are greater than all other indicators, indicating that the CR_FORM is the most critical factor affecting the severity of a crash. In addition, the regression coefficient of RD_AS is 1.136, indicating that the probability of a serious crash with access segment is greater. As far as VEH_TYPE is concerned, the regression coefficient of crash vehicles involving motorcycles is 0.963 , the regression coefficients is smaller than DR_BE, CR_FORM, and RD_AS but larger than DR_BE, DR_LO, and EN_TA, respectively, and the results show that the vehicle type is the key factor affecting the severity of the crash on two-lane rural roads. The regression coefficient of DR_LO is -0.298 , to indicate that that the local driver will cause a more serious crash. The regression coefficient for EN_TA is -0.220 , which means that the probability of a serious crash on working days is less than that of the week day.

\section{Discussion}

5.1. Cause Consequence Analysis. The effect of changes on dependent variables for significant variables was further analysed. Based on the estimation results of the randomparameter ordered probit model, the average pseudoelasticity of 7 significant variables is calculated according to formulae (5) and (6), to explain the direction and strength of the influence of related factors on China two-lane road crash severity. The marginal effects of each significant variable are computed as shown in Table 7. The effects of these significant contributing factors are further discussed.

5.1.1. Driver Attribution. From the analysis of whether the local or nonlocal driver presents significant differences in influencing the crash severity, it was found that there exist significant differences between local and nonlocal drivers in minor collision, serious collision, and malignant collision. 
TABLE 5: Goodness-of-fit measures for fixed- and random-parameter ordered probit models.

\begin{tabular}{|c|c|c|}
\hline Model statistics & Fixed-parameter ordered probit model & Random-parameter ordered probit model \\
\hline Number of observations, $n$ & 1049 & 1049 \\
\hline Number of parameters, $K$ & 17 & 7 \\
\hline AIC & 2198.80 & 2158.20 \\
\hline Log-likelihood & -1080.42 & -1066.12 \\
\hline Degrees of freedom & & \\
\hline$x^{2}$ & & \\
\hline$P$ value & & \\
\hline
\end{tabular}

TABLE 6: Estimation results for the random-parameter ordered probit model.

\begin{tabular}{|c|c|c|c|c|c|c|}
\hline Variables & Mean & Standard error & $Z$ & Prob. $\mathrm{z}>Z^{*}$ & Prob. $\leq 0)(\%)$ & Prob. $>0(\%)$ \\
\hline Constant & 0.045 & 0.173 & 0.260 & 0.793 & 39.74 & 60.26 \\
\hline DR_LO & $-0.29817^{* * *}$ & 0.086 & -3.470 & 0.001 & 4.81 & 95.19 \\
\hline DR_BE & $-0.49270^{* * *}$ & 0.083 & -5.910 & 0.000 & 1.81 & 98.19 \\
\hline$D R \_B E$ & $1.24031^{* * *}$ & 0.078 & 15.810 & 0.000 & 0.99 & 99.01 \\
\hline RD_AS & $-0.42897^{* * *}$ & 0.095 & -4.530 & 0.000 & 4.79 & 95.21 \\
\hline$R D \_A S$ & $1.13642^{* * *}$ & 0.093 & 12.190 & 0.000 & 0.99 & 99.01 \\
\hline EN_TA & $-0.22026^{* * *}$ & 0.084 & -2.620 & 0.009 & 0.44 & 99.56 \\
\hline CR_FORM2 & $0.17047^{*}$ & 0.095 & 1.800 & 0.071 & 3.59 & 96.41 \\
\hline CR_FORM3 & $-1.18383^{* * *}$ & 0.224 & -5.270 & 0.000 & 1.81 & 98.19 \\
\hline CR_FORM3 & $1.13642^{* * *}$ & 0.093 & 12.190 & 0.000 & 0.99 & 99.01 \\
\hline VEH_TYPE3 & $0.96320^{* * *}$ & 0.163 & 5.920 & 0.000 & 1.81 & 98.19 \\
\hline
\end{tabular}

Note. The italicized ones represent estimates for the variables resulting in random parameters. ${ }^{*} P<0.1,{ }^{* *} P<0.05$, and ${ }^{* * *} P<0.001$.

As illustrated in Tables 6 and 7, the crash frequency of the local driver is higher than the nonlocal driver. The parameter of this variable is normally distributed with mean -0.29 and standard deviation 0.086 . It means $95.19 \%$ of them tend to sustain higher injury. Per the marginal effects, the nonlocal driver decreases the probability of serious and malignant injury by $46.82 \%$ and $125.65 \%$, respectively, and this finding is supported by conclusions from previous studies that local drivers are more frequently involved in fatal crashes [32]. This interesting result could be attributed to the fact that nonlocal drivers are not familiar with the road conditions of this two-lane rural road, including the setting of traffic signs and signals. Therefore, nonlocal drivers choose to drive cautiously at low speeds and pass after observation. However, for local drivers, they drive on this road every day and think they are quite familiar with the road conditions, so they do not have the consciousness of slowing down ahead of time, and there is no time to avoid escaping the probability of serious collisions when a dangerous situation occurs.

5.1.2. Driving Behaviours. Regarding the indicator of driving behaviour, there is a higher probability of serious injury when high-risk driving behaviour occurs under two-lane rural road driving conditions. To be more precise, it can be seen from the parameter distribution shown in Table 7 that compared with no high-risk driving behaviour, the driver adopts high-risk driving behaviour to drive on two-lane rural roads, and it increases the likelihood of more severe crashes. That is, once a crash occurs, the probability of causing serious and malignant injuries increases by $77.81 \%$ and $239.25 \%$, respectively.
That can be interpreted that drivers will be more dangerous with high-risk driving behaviours under unfavourable alignment conditions, such as fatigue driving, speeding, converse driving, and aggressive driving, and eventually increase the probability of being severely and fatally injured in traffic accidents. This is a consistent result also can be found in the study by Kim et al. [30], which suggested the severity of this higher risk-taking behaviour increases.

5.1.3. Access Segment. From the perspective of the location of the crash, the two indicators of the access segment and the village segment are the characteristic indicators which proposed to measure the crash severity, based on the actual characteristics of rural roads in China. However, according to the model estimation results (shown in Table 6) and the marginal effect results (shown in Table 7), it can be found that the indicator of the access segment has negative effect on the latent severity propensity but is significant $(P$ value less than 0.001).

Another concern is that access segment is a heterogeneous variable. The result points out that $99.01 \%$ cyclists tend to suffer higher injury if they involve nonaccess segment. In Table 7, the results of marginal effects indicate that crashes occurring in road access segment decrease the chance of minor collision. And the probability of serious collision and malignant collision in access segments decreased by $66.03 \%$ and $165.61 \%$, respectively. This interesting result could be partially explained by the fact that, due to the large proportion of access segments in China rural roads, and the lack of isolation facilities at the access segment, pedestrians, vehicles, and animals often cross the road, 
creating a complex traffic flow environment. The driving environment of two-lane rural roads is quite dangerous. However, Chinese drivers are more sensitive to this danger perception and drive more cautiously, so they can reduce the severity of the crash.

5.1.4. The Day of Weekday. In relation to the time of the week, as analysed before, it has been concluded that the day of week is significantly related to the crash severity. Furthermore, comparing and analysing the results of the marginal effects of time of the week on the crash severity at different levels (shown in Table 7), it is easy to obtain this result that a crash occurs on a working day instead of weekend or holiday, the probability of a minor collision will increase by $10.47 \%$, and the probability of serious and vicious collision will decrease by $34.89 \%$ and $96.79 \%$ respectively, which is supported by conclusions from previous studies [33].

There is a reasonable explanation is that two-lane rural roads are the key roads connecting country towns and counties, and students or employed people in county towns usually choose to return to their villages or towns on Friday nights and then return to their schools or workplaces on Sunday, which leads to a significant increase in traffic flows on weekends or holidays, further increases the exposure of passenger vehicles in the two-lane rural roads; this exposure signifies greater interaction between passenger vehicles and others, which may increase the probability of severe crashes. As a consequence, in contrast, the times of weekends or holidays increase the likelihood of serious or malignant collisions.

5.1.5. Crash Form. Regarding the collision forms in rural two-lane road conditions, two kinds of crash form cause higher injury: vehicle-vehicle collision and vehicle-fixed object crashes. The effect of crash form on crash severity might be different, the vehicle-vehicle collision is positively associated with the increased levels of crash injury severity, vehicle-fixed object crashes play a negative role in two-lane rural road' crash injury with different magnitude. Per the marginal effects as shown in Table 7 , vehicle collisions are found as the most significant collision type, increasing the probability of higher injury severity level on two-lane rural roads. To be more precise, the positive elasticity of the vehicle collision and specific to serious and malignant collisions indicates that as a vehicle collides with a moving vehicle, collision outcomes of two-lane rural crashes are more likely to be serious and malignant rather than minor; this is likely because drivers may have less reaction time and perception ability on crash risk of moving vehicle on the road.

Table 7 shows that it decreases the probability of minor collision by $8.38 \%$ and increases the probability of serious and malignant injuries by $27.81 \%$ and $96.63 \%$, respectively. This finding suggests that crash form has significant impact on the crash severity, and these results are consistent with previous studies. On the other hand, in contrast to vehiclevehicle collision, the vehicle-fixed object crashes occurring in rural areas were found to decrease the probability of serious and malignant injuries by $137.87 \%$ and $194.88 \%$, respectively, and decrease the probability of minor injury by $41.10 \%$. One possible reason is that due to the complex linear conditions of rural roads, the proportion of protective structures such as cement concrete safety guard bar and corrugated beam steeled protection fence is higher, and it plays an important role in preventing more serious crash such as falling off cliffs when a vehicle collides.

5.1.6. Vehicle Type. Whether passenger car, truck, and motorcycle present significant difference in effecting the severity of the crash is analysed. The impact of different types of involved vehicles on crash severity was investigated in our study, and the result is shown in Table 7 . It was found that there exist significant differences between passenger cars and trucks, and collisions involving trucks or motorcycles significantly increase the likelihood of a fatal accident and greater than the likelihood of a fatal crash only involved passenger cars. This result is consistent with the previous finding that accidents involving motorcycles are more severe than those involving passenger cars [31].

To be more precise, the most serious collisions involve unprotected vehicles or trucks on rural roads. The probability of serious and malignant accidents involving trucks or motorcycles will increase by $153.12 \%$ and $1690.34 \%$, respectively, when compared to only passenger cars involved, which is slightly different from other types of crash vehicles on different road types. Light and convenient motorcycles have become the most common means of personal travel in rural roads, but the stability of motorcycles is obviously less than cars, and motorcycle drivers have been exposed to the car in the event of a crash; motorcycle drivers are surely more vulnerable to malignant injuries. On the other hand, clearly, truck mechanical and loading conditions are compelling issues, significantly related to serious and malignant collisions upon crash occurrence.

5.2. The Influence Mechanism of Significant Variables Obtained. Since the coefficients estimated from the random-parameter ordered model cannot be directly interpreted as the influence of risk factors, the marginal effect value is used to describe the impact of significant variables on the severity of collisions at different levels, as shown in Figure 6. Figure 6 shows a summary of the main results of the analysis of the crash severity in China rural roads. It contains nine significant factors in driver, vehicle, road, environment, and crash. The red arrow indicates a negative effect on the severity of the crash, on the contrary, the black arrow indicates a positive influence, and the pyramid represents three levels of the severity of the accident.

As shown in this figure, "accident form" and "vehicle type" are the most important factors affecting the crash severity of rural road. The results of this study suggest that these factors need to be taken into account in the formulation of rural road traffic safety management policies. Secondly, from the driver's perspective, the driver's 


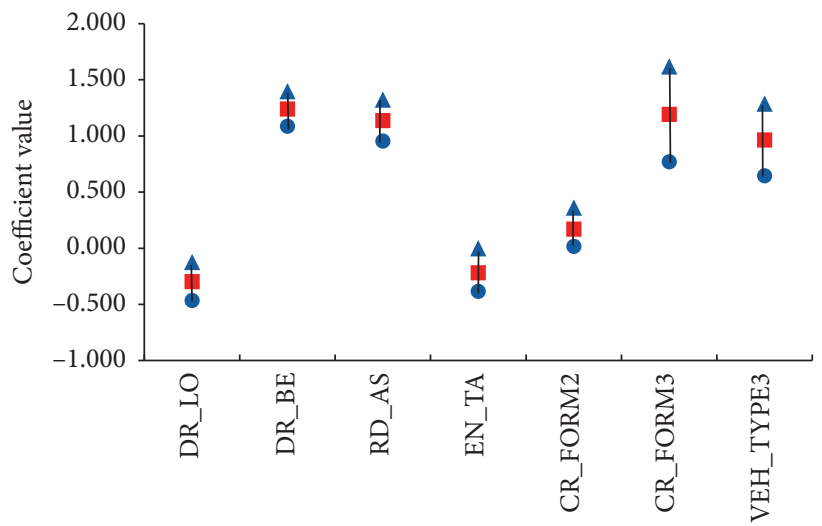

Significant variable

- Coefficients

- $97.50 \%$

\ $2.50 \%$

FIgURE 5: The distribution of coefficients.

TABLe 7: Marginal effects and elasticities.

\begin{tabular}{lcccccc}
\hline \multirow{2}{*}{ Significant variable } & \multicolumn{3}{c}{ Margin effect } & & \multicolumn{2}{c}{ Elasticity } \\
& Minor collision & Serious collision & Malignant collision & Minor collision & Serious collision & Malignant collision \\
\hline DR_LO & 0.108 & -0.107 & -0.002 & $14.05 \%$ & $-46.82 \%$ & $-125.65 \%$ \\
DR_BE & -0.180 & 0.177 & 0.003 & $-23.40 \%$ & $77.81 \%$ & $239.25 \%$ \\
RD_AS & 0.153 & -0.151 & -0.002 & $19.80 \%$ & $-66.03 \%$ & $-165.61 \%$ \\
EN_TA & 0.081 & -0.080 & -0.001 & $+10.47 \%$ & $-34.89 \%$ & $-96.79 \%$ \\
CR_FORM2 & -0.065 & 0.063 & 0.001 & $-8.38 \%$ & $27.81 \%$ & $96.63 \%$ \\
CR_FORM3 & 0.317 & -0.314 & -0.002 & $41.10 \%$ & $-137.87 \%$ & $-194.88 \%$ \\
VEH_TYPE3 & -0.370 & 0.349 & 0.020 & $-47.96 \%$ & $153.12 \%$ & $1690.34 \%$ \\
\hline
\end{tabular}

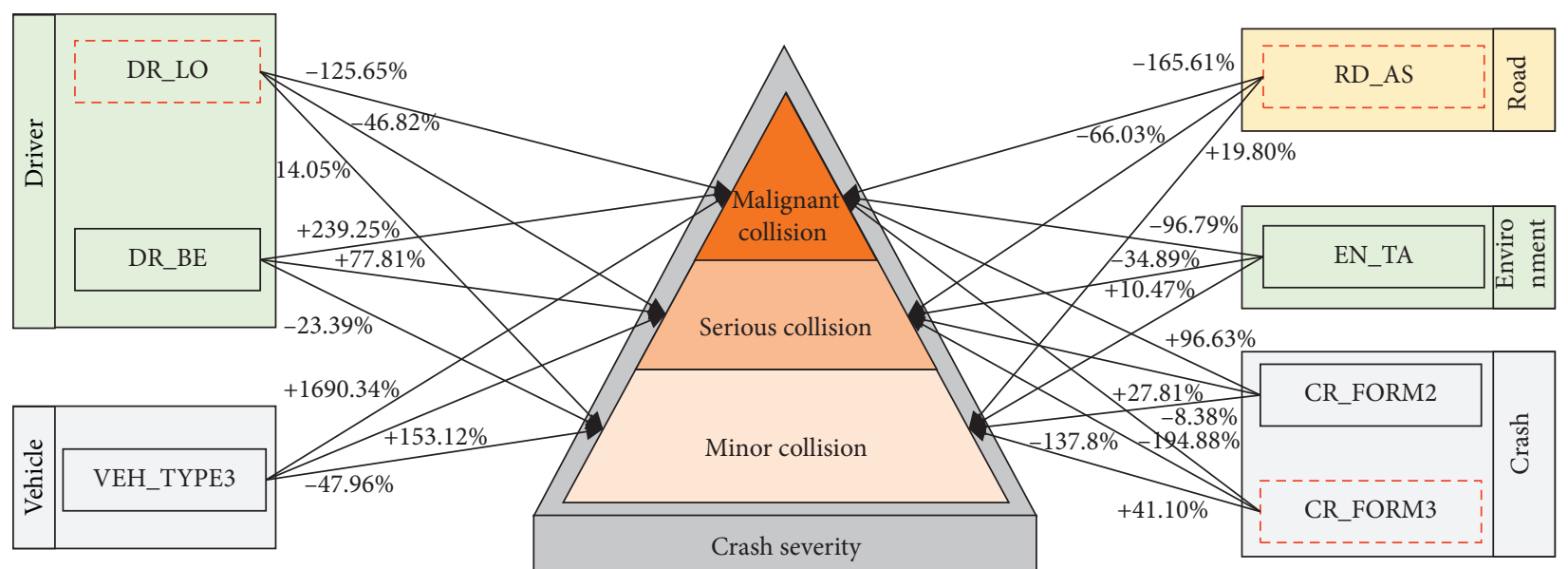

FIGURE 6: The influence mechanism of significant variables obtained.

attribution and driving behaviour also play a fundamental role in the severity of the crash. In addition, road access segments and holidays are also important for the crash severity, in which case the impact of road access and holidays on accidents needs to be considered and management measures developed.
The results and conclusions of the study analysis can help traffic safety management managers to design management policies and accident prevention measures to provide a theoretical basis, especially for specific types of rural roads and user categories. Understanding what is important and to what extent they affect the severity of accidents is a 
fundamental pillar for better understanding and addressing existing rural road safety issues.

\section{Conclusions}

This study focuses on the effect of various factors on the crash severity of two-lane rural roads, specific focus was placed on exploring the role of heterogeneity and the potential sources of heterogeneity regarding their impacts on crash injury severity outcomes, and it aims to provide a reasonable reference for the formulation and management policy of two-lane rural roads, safety operations, and traffic accident prevention countermeasures to ensure driving safety as much as possible and reduce the collision rate and severity of two-lane rural roads. $t$. To account for the ordinal nature of response outcomes and unobserved heterogeneity, this paper divides the crash severity into three levels: minor, serious, and malignant collisions. A random-parameter ordered probit model was adopted to identify risk factors. According to the record of 1490 crashes occurring on twolane rural roads in China during the past 6 years. From the perspective of sustainable development, the impact of drivers, vehicles, roads, environment, and accidents on the severity of rural road crash is comprehensively considered. With the seriousness of crash used as a dependent variable, fourteen influencing factors (except dummy variables) on crash severity were selected, which include driver gender, age, attribution, driving behaviour, vehicle type, road horizontal alignment, road vertical alignment, access segment, village segment, light, weather, road surface, the day of week, and crash form, and exploring the influence of contributing factors on the crash severity.

In order to capture underlying unobserved characteristics, the crash injury severity of the random-parameter ordered probit model was established, and the regression models obtained satisfy the likelihood ratio test, meeting the significance test and indicating that the random-parameter ordered probit model can effectively analyse the influencing factors of the severity of two-lane rural crashes. The model results show that six variables of crash form, driver attribution, illegal driving behaviour, vehicle type, access segment, and holidays have a significant impact on the crash severity of two-lane rural roads, and the impacts of driving behaviours, access segment, and vehicle-fixed object crashes belong to random parameters; this indicator had significant variation across observations. Among the significant variables obtained, the crash form, vehicle type, holiday, and illegal driving behaviour have positive impact on the crash severity. On the contrary, the driver's attribution and access segment have negatively impact.

The results of elastic analysis of significant variables show that, compared with the nonlocal driver, the local driver indicator has higher probability of severe accidents. As for the driving behaviour, it is closely related with twolane rural road crashes. The high-risk behaviour of drivers leads to the increase of likelihood of serious or malignant collisions. Hence, the rural road transportation management department should strictly require the driver's skills and operations and conduct safety education and training to the driver. Besides, when crashes occur on a nonaccess segment, a higher probability of serious or vicious collisions appeared on two-lane rural roads. As far as holidays are concerned, due to the increase in traffic flow and the decrease in management inspectors, the traffic state of the two-lane rural road is not fully controlled. As a result, most drivers lax thinking, and it is easy to violate traffic rules, which aggravated the crash severity. Therefore, the transportation department should strengthen the management and punishment during the holidays and recommend that drivers choose low traffic travel time to reduce the severity of collisions.

Among the vehicle types identified, the vehicle types do show an obvious correlation with crash injury severity, especially involved motorcycle, to influence crash injury severity most. To reduce the severity of the crash, the driver's safety awareness should be improved for motorcycle drivers and they should wear helmets and drive safely in accordance with regulations. In comparison with other crash forms, the vehicle-vehicle collision is more likely to lead to severe injury collisions. To prevent and reduce the occurrence of serious crash, the driver should drive at the prescribed speed and actively maintain a safe driving distance from the other vehicle for emergency braking.

The research in this paper can provide a theoretical basis for the traffic safety management department to formulate accident prevention countermeasures. However, there are two main limitations: (1) the crash data were obtained from the local highway bureau and may thus be subject to some degree of inadequacies (e.g., the possibility of underreporting of less severe crashes and the unavailability of relevant variables), more attention should be paid to advocate the integration of police-reported data and other data sources in the analysis of crash severity (e.g., questionnaire surveys, field observations, and driving simulations), and this is one of directions for our future work. (2) This study retained a sample of 1,490 crashes (i.e., 66.31\%) in the basic database of the two-lane rural roads for further analysis, and crash records with complete information are more likely to be those resulting in fatal and serious injuries, inducing the risk of biased sampling. In future research, more comprehensive data across multiple years are recommended for indepth research on two-lane rural roads, to achieve a more explicit understanding of the influence mechanism underlying crash injury severity on the two-lane rural roads.

\section{Data Availability}

The data used to support the findings of this study are available from the corresponding author upon request.

\section{Conflicts of Interest}

The authors declare that they have no conflicts of interest.

\section{Acknowledgments}

This research was jointly supported by the National Key R\&D Programme of China (no. 2017YFC0803906), the 
National Natural Science Foundation of China (52062024 and 52002161), the General Programme of Natural Science Foundation, Yunnan Province, China (Grant No. 2019FB072) and the National Engineering Laboratory Open Research Fund Project for Land Traffic Meteorological Disaster Prevention and Control Technology of China (NEL-2019-05).

\section{References}

[1] Traffic Management Bureau of the Ministry of Public Security, Road Traffic Accident Statistic Annual Report of the People's Republic of China, Traffic Management Research Institute of the Ministry of the Public Security, Wuxi, China, 2014.

[2] S. Cafiso, A. Di Graziano, G. Di Silvestro, G. La Cava, and B. Persaud, "Development of comprehensive accident models for two-lane rural highways using exposure, geometry, consistency and context variables," Accident Analysis \& Prevention, vol. 42, no. 4, pp. 1072-1079, 2010.

[3] NHTSA, Rural/Urban Comparison of Traffic Fatalities, National Highway Traffic Safety Administration, Washington, DC, USA, 2017.

[4] J. Xu, W. Lin, X. Wang, and Y. M. Shao, "Acceleration and deceleration calibration of operating speed prediction models for two-lane mountain highways," Journal of Transportation Engineering, Part A: Systems, vol. 143, pp. 1-13, Article ID 4017024, 2017.

[5] P. T. Savolainen, F. L. Mannering, D. Lord, and M. A. Quddus, "The statistical analysis of highway crash-injury severities: a review and assessment of methodological alternatives," Accident Analysis \& Prevention, vol. 43, no. 5, pp. 1666-1676, 2011.

[6] P. Kopelias, F. Papadimitriou, and K. Papandreou, "Urban freeway crash analysis: geometric, operational, and weather effects on crash number and severity," Transportation Research Record Journal of the Transportation Research Board, vol. 2015, pp. 123-131, 2007.

[7] R. Tay and S. M. Rifaat, "Factors contributing to the severity of intersection crashes," Journal of Advanced Transportation, vol. 41, no. 3, pp. 245-265, 2007.

[8] C. Caliendo, M. L. De Guglielmo, and M. Guida, "Comparison and analysis of road tunnel traffic accident frequencies and rates using random-parameter models," Journal of Transportation Safety \& Security, vol. 8, no. 2, pp. 177-195, 2016.

[9] Y. C. Chiou, C. C. Hwang, C. C. Chang, and C. Fu, "Reprint of modeling two-vehicle crash severity by a bivariate generalized ordered probit approach," Accident Analysis and Prevention, vol. 61, pp. 94-106, 2013.

[10] R. Yu and M. Abdel-Aty, "Using hierarchical bayesian binary probit models to analyze crash injury severity on high speed facilities with real-time traffic data," Accident Analysis \& Prevention, vol. 62, pp. 161-167, 2014.

[11] E. R. Rusli, M. M. Haque, M. J. King, and S. V. Wong, "A comparison of road traffic crashes along mountainous and non-mountainous roads in Sabah, Malaysia," in Proceedings of the Australasian Road Safety Conference, pp. 14-16, Gold Coast, Australia, October 2015.

[12] F. L. Mannering, V. Shankar, and C. R. Bhat, "Unobserved heterogeneity and the statistical analysis of highway accident data," Analytic Methods in Accident Research, vol. 11, pp. 1-16, 2016.
[13] F. L. Mannering and C. R. Bhat, "Analytic methods in accident research: methodological frontier and future directions," Analytic Methods in Accident Research, vol. 1, pp. 1-22, 2014.

[14] X. Zhu and S. Srinivasan, "A comprehensive analysis of factors influencing the injury severity of large-truck crashesfluencing the injury severity of large-truck crashes," Accident Analysis \& Prevention, vol. 43, no. 1, pp. 49-57, 2011.

[15] C. Lee and X. Li, "Analysis of injury severity of drivers involved in single-and two-vehicle crashes on highways in Ontario," Accident Analysis \& Prevention, vol. 71, pp. 286295, 2014.

[16] B. Peterson and F. E. Harrell, "Partial proportional odds models for ordinal response variables," Applied Statistics, vol. 39, no. 2, pp. 205-217, 1990.

[17] P. Michalaki, M. A. Quddus, D. Pitfield, and A. Huetson, "Exploring the factors affecting motorway accident severity in England using the generalised ordered logistic regression modelffecting motorway accident severity in England using the generalised ordered logistic regression model," Journal of Safety Research, vol. 55, pp. 89-97, 2015.

[18] J. D. Lemp, K. M. Kockelman, and A. Unnikrishnan, “Analysis of large truck crash severity using heteroskedastic ordered probit models," Accident Analysis \& Prevention, vol. 43, no. 1, pp. 370-380, 2011.

[19] S. Yasmin and N. Eluru, "Evaluating alternate discrete outcome frameworks for modeling crash injury severity," Accident Analysis \& Prevention, vol. 59, pp. 506-521, 2013.

[20] M. Abdel-Aty, "Analysis of driver injury severity levels at multiple locations using ordered probit models," Journal of Safety Research, vol. 34, no. 5, pp. 597-603, 2003.

[21] Q. Wu, F. Chen, G. Zhang, X. C. Liu, H. Wang, and S. M. Bogus, "Mixed logit model-based driver injury severity investigations in single- and multi-vehicle crashes on rural two-lane highways," Accident Analysis \& Prevention, vol. 72, pp. 105-115, 2014.

[22] H. Zhou, C. Yuan, N. Dong, S. C. Wong, and P. Xu, "Severity of passenger injuries on public buses: a comparative analysis of collision injuries and non-collision injuries," Journal of Safety Research, vol. 74, pp. 55-69, 2020.

[23] F. Chen, M. T. Song, and X. X. Ma, "Investigation on the injury severity of drivers in rear-end collisions between cars using a random parameters bivariate ordered probit model," International Journal of Environmental Research and Public Health, vol. 16, pp. 1-12, 2019.

[24] M. G. H. Bell, "Future directions in traffic signal control," Transportation Research Part A: Policy and Practice, vol. 26, no. 4, pp. 303-313, 1992.

[25] K. Yunxia, "Safety characteristics of two-lane highway sections passing through towns/villages in mountainous area based on negative binomial prediction model," Journal of Highway and Transportation Research and Development, vol. 29, pp. 110-115, 2012.

[26] A. M. Sadri, S. V. Ukkusuri, and P. Murray-tuite, "A random parameter ordered probit model to understand the mobilization time during hurricane evacuation," Transportation Research Part C: Emerging Technologies, vol. 32, pp. 21-30, 2013.

[27] S. Mokhtarimousavi, J. C. Anderson, A. Azizinamini, and M. Hadi, "Factors affecting injury severity in vehicle-pedestrian crashes: a day-of-week analysis using random parameter ordered response models and artificial neural networks," International Journal of Transportation Science and Technology, vol. 9, no. 2, pp. 100-115, 2020. 
[28] S. Washington, M. Karlaftis, and F. Mannering, Statistical and Econometric Methods for Transportation Data Analysis, CRC Press, Boca Raton, FL, USA, 1st edition, 2003.

[29] J.-K. Kim, S. Kim, G. F. Ulfarsson, and L. A. Porrello, "Bicyclist injury severities in bicycle-motor vehicle accidents," Accident Analysis \& Prevention, vol. 39, no. 2, pp. 238-251, 2007.

[30] J.-K. Kim, G. F. Ulfarsson, S. Kim, and V. N. Shankar, "Driverinjury severity in single-vehicle crashes in California: a mixed logit analysis of heterogeneity due to age and gender," Accident Analysis \& Prevention, vol. 50, pp. 1073-1081, 2013.

[31] Q. Zeng, W. Hao, J. Lee, and F. Chen, "Investigating the impacts of real-time weather conditions on freeway crash severity: a bayesian spatial analysis," International Journal of Environmental Research and Public Health, vol. 17, no. 8, p. 2768,2020 .

[32] S. D. Chen, S. W. Zhang, Y. Y. Xing, and J. Lu, "Identifying the factors contributing to the severity of truck-involved crashes in shanghai river-crossing tunnel," International Journal of Environmental Research and Public Health, vol. 17, no. 3155, 2020.

[33] Y. Wang and C. G. Prato, "Determinants of injury severity for truck crashes on mountain expressways in China: a case-study with a partial proportional odds model," Safety Science, vol. 117, pp. 100-107, 2019. 